\title{
A Time-Domain Method for the Analysis of Thermal Impedance Response Preserving the Convolution Form
}

\author{
Manuel Carmona, Santiago Marco, Jordi Palacín, and Josep Samitier
}

\begin{abstract}
The study of the thermal behavior of complex packages as multichip modules (MCM's) is usually carried out by measuring the so-called thermal impedance response, that is: the transient temperature after a power step. From the analysis of this signal, the thermal frequency response can be estimated, and consequently, compact thermal models may be extracted. We present a method to obtain an estimate of the time constant distribution underlying the observed transient. The method is based on an iterative deconvolution that produces an approximation to the time constant spectrum while preserving a convenient convolution form. This method is applied to the obtained thermal response of a microstructure as analyzed by finite element method as well as to the measured thermal response of a transistor array integrated circuit (IC) in a SMD package.
\end{abstract}

Index Terms - Compact models, deconvolution, METS, packaging, thermal impedance, thermal model, thermal resistance, thermal transients.

\section{INTRODUCTION}

$\mathbf{M}$ OST thermal studies of electronic systems are carried out in steady-state conditions and the thermal performance is described by a single parameter: the junctionto-case (or junction-to-ambient) thermal resistance. However, in several areas of application the importance of the dynamic behavior is arising. In the search for low power designs in portable systems, components will have to work in thermal transient conditions to save battery power [1]. On the other hand, microsystems technologies are bringing new devices whose principle of operation is based on thermal dynamics [2], [3].

These considerations show the need of compact thermal models that permits the accurate prediction of the thermal transient behavior of chip packages to nonsteady or cyclic powering conditions, and thermally based microsensors or actuators. Such models can be included in high level simulators and described in analog hardware description languages per-

Manuscript received September 29, 1998; revised March 5, 1999. This paper was recommended for publication by Editor B. Courtois upon evaluation of the reviewers' comments. This work was presented at the Fourth International Workshop on Thermal Investigations of IC's and Microstuctures, Cannes, "Côte d'Azur," France, September 27-29, 1998. This work was supported by Spanish CICYT TIC98-0987-C03-03.

M. Carmona, S. Marco, and J. Samitier are with the Electronics Department, Universitat de Barcelona, Barcelona 08028, Spain.

J. Palacín is with the Computer and Industrial Engineering Department, Universitat de Lleida, Lleida 25001, Spain.

Publisher Item Identifier S 1521-3331(99)05394-5. mitting the easy simulation of thermally based microsystems or electrothermal interactions [4].

The extraction of such kind of models is not trivial because of the three-dimensional (3-D) and distributed character of the problem. This can cause the appearance of continuous time constant distributions hindering the extraction of a lumped model [5].

Moreover, the thermal dynamics of the package can give us additional insight about its internal construction. Even, diagnostics can be extracted from the analysis revealing the presence of defects as delamination [6].

Among the different alternatives to extract the thermal dynamic behavior of a package, the analysis of the thermal impedance response has become a common choice [7]. The thermal impedance response can come, either from experimental measurements, or from physical simulation using 3-D solvers based in finite element, finite differences or alternative methods. In the 1st case, however, noise is a main concern.

The thermal impedance response usually consists in a superposition of exponential decays. The analysis of these signals is extremely sensitive to noise and truncation since real exponentials, in contrast to complex exponentials, do not form an orthogonal basis of functions in the real axis.

To obtain the final thermal compact model representing the package thermal dynamics it is necessary to approximate the multiexponential decay with the minimum number of exponential terms to avoid unnecessary model complexity. Once the underlying time-constants and their amplitudes have been determined, the compact thermal model can be determined. It is usually an RC network in which the resistances are a lumped representation of the distributed 3-D thermal resistance between the junction and the case. Capacitances are a lumped representation of the 3-D thermal-mass distribution. The analysis of the thermal transient leads directly to the Foster equivalent network representation. As this network has not a physical interpretation, a Foster-to-Cauer network transformation [8] is necessary (Fig. 1).

To analyze the thermal transient, some authors have proposed direct nonlinear least squares fit [9], although this method has been shown not to be very accurate [10]. It is also possible to differentiate the transient to obtain the impulse response, apply a discrete Fourier Transform and finally apply a nonlinear fit in the frequency domain [1]. Szekely differentiates the thermal impedance response in the logarithm of the time and then applies a deconvolution method [11]. 


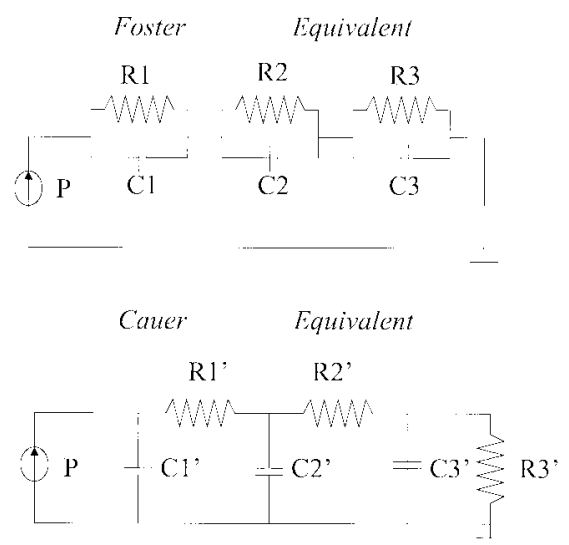

Fig. 1. Foster and Cauer RC networks representing the thermal models.

The purpose of this work is to present an extension of a method proposed by Szekely [11] with improved timeresolution in the extraction of discrete time-constant distributions while preserving a convolution form. We have named such method multi-exponential transient spectroscopy (METS). The theoretical basis of the method will be presented in Section II. In Section III, this method will be applied to a thermo-pneumatic micropump to extract a linear transfer function representing in a compact form the dynamic thermal behavior. As a second example, the method will be applied to the thermal analysis of a transistor array IC in a $16 \mathrm{Ld}$ small outline plastic package (SOIC): HFA3096. Finally, Section IV will summarize the main conclusions.

\section{THEORY}

\section{A. Thermal Impedance Response}

The dynamic behavior of a linear system is represented in the time domain by its impulse response, whose Fourier transform is the frequency response of the system. Throughout this paper, we assume that the systems are sufficiently linear under the operation conditions. This means, in particular, that we can neglect the nonlinearities introduced by the temperature dependence of the thermal conductivity and the contribution of radiation to the total heat dissipation is small. In thermal systems, the usual way to characterize the system dynamics is the analysis of the thermal response to a step in the applied power. The so-called transient thermal impedance is given by

$$
Z_{\mathrm{th}}(t)=\frac{T(t)-\text { tref }}{P}
$$

where $T(t)$ is the temperature at a certain point of interest, $T_{\text {ref }}$ is a reference temperature (usually ambient), and $P$ is the amplitude of the power step. So we consider that the system input is a power signal, while the output is a temperature. The thermal impedance response is usually plotted in the logarithm of time because of the presence of time constants that can differ by several orders of magnitude. In addition, and as mentioned in the introduction, the thermal impedance response gives an insight about the thermal resistance path. This is because larger time constants are associated to structures with larger heat capacitance that are far from the sensing location (substrate, package ...).

A complete description of the thermal dynamics is the socalled time-constant spectrum. The system transfer function can be written as (lumped element approximation)

$$
H(s)=\sum_{i=1}^{M} \frac{A_{i}}{s \tau_{i}+1} .
$$

where $A_{i}$ is the exponential term amplitude and $\tau_{i}$ the corresponding time-constant. In this way, the sum of amplitudes is the steady-state thermal resistance. We call the time constant spectrum a graphical representation of the pairs $\left(\tau_{i}, A_{i}\right)$ in the logarithm of time. It is clear that this set of pairs provides a complete parametric representation of the system transfer function. The analysis of the thermal impedance response can provide this representation which is the base for thermal compact modeling, diagnostics, etc.

\section{B. METS Method}

The analysis of a multiexponential transient can be carried out by differentiation in the logarithm of the time

$$
\begin{aligned}
y(t) & =\sum_{i=1}^{M} A_{i}\left(1-e^{-t / \tau_{i}}\right) \\
M_{1}(z) & =\frac{d y(z)}{d z}=\int_{-\infty}^{+\infty} R(\mu) h_{1}(z-\mu) d \mu
\end{aligned}
$$

where $z=\ln t, h_{1}(z)=\exp (z-\exp (z))$ and $R(z)$ is the signal containing the information of the time-constant spectrum. So $R(z)$ can be obtained by deconvolution. This problem is ill-conditioned since $h_{1}(z)$ acts as a low-pass filter. Direct Fourier inverse filtering amplifies high frequency noise although this problem can be mitigated with appropriate filtering of $M_{1}(z)$. This procedure was originally proposed by Gardner [12], further explored by Smith [13] and recently applied to thermal response by Szekely [14]. The authors have also proposed the use of nonlinear iterative deconvolution methods as the Jansson's and Gold's method [15]. With these methods resolutions of 1.25 octaves have been achieved with initial noise rms values of $1 \mathrm{mV}$ in $1 \mathrm{~V}$ transients [16]. The truncated Van-Citter [15] method will be used in this work as a reference technique for comparison purposes. This iterative method can be used for deconvolution

$$
R^{k+1}=p R^{k}+b\left(y-p h_{1} * R^{k}\right)
$$

where

$R^{k} k$ th estimate of $R(z)$;

$b$ constant;

$k$ iteration number;

$p$ nonlinear constraint that limits the values that $R^{k}$ can achieve and it truncated artifacts that may appear during the iterations.

The algorithm initialization is

$$
R^{0}=y
$$




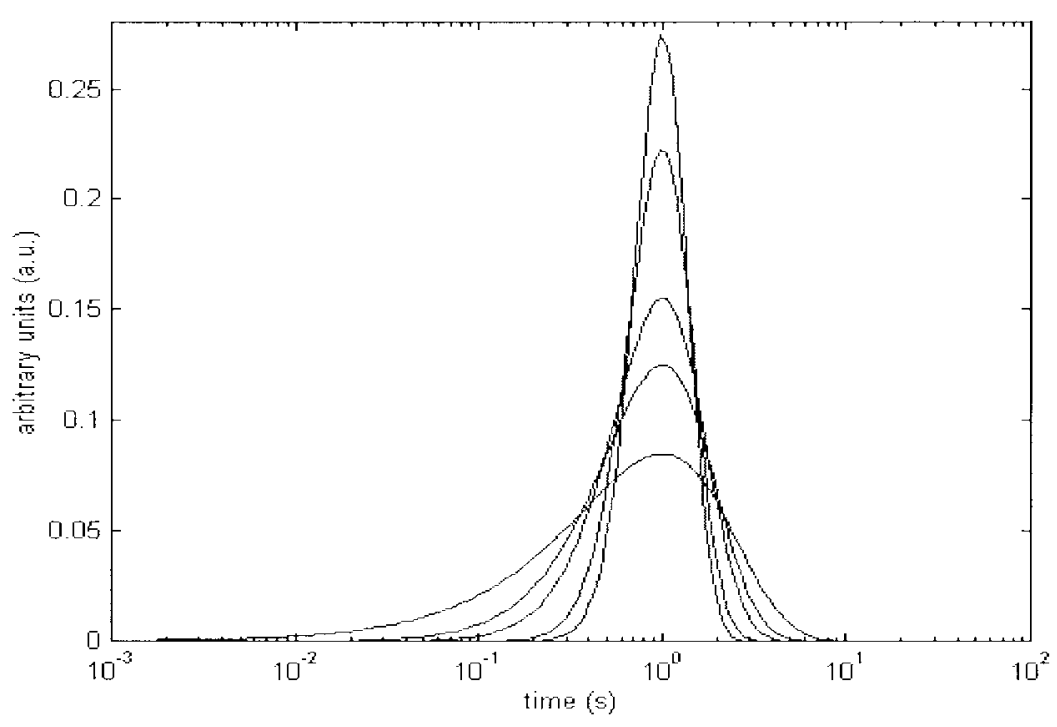

Fig. 2. METS kernels order 1, 2, 3, 6, and 9.

The iterations are carried out until a given error criterion is satisfied. The parameter $b$ is selected to achieve a rate of convergence and it depends on the class of data.

However, the authors experience indicates that although deconvolution methods can offer great increases in resolution [compared to 3.5 octaves in $M_{1}(z)$ ], the peaks position and amplitude suffer accuracy losses.

Here we will present a method that combines an iterative deconvolution method that preserves the convolution form of $M_{1}(z)$. The method defines a family of kernels given by $h_{n}(z)=\exp (n z-\exp (z))$ [17] and the METS signals are

$$
M_{n}(z)=\int_{-\infty}^{+\infty} R(\mu) h_{n}(z-\mu) d \mu
$$

Differentiation of the convolution product permits us to observe that the $M_{n}(z)$ can be obtained from lower orders by the following recurrence formula:

$$
M_{n+1}(z)=n M_{n}(z)-\frac{d M_{n}(z)}{d z} .
$$

The key point in this procedure is the resolution increase obtained, because the full width half maximum of the kernels decreases as $n$ increases (Fig. 2).

The final value of the parameters can be obtained by a constrained nonlinear fit because of the known shape of the convolution kernel. For details of the discrete time-implementation see [16], [17].

As already mentioned, every estimation method is finally limited by the noise level present in the original signal. In our method, the iterations are limited by high frequency noise amplification due to the differentiation step included in the recurrence formula. We have calculated the signal degradation involved in this method. The result shows how the amplitude to noise ratio worsens with the iterations. Despite of that, a

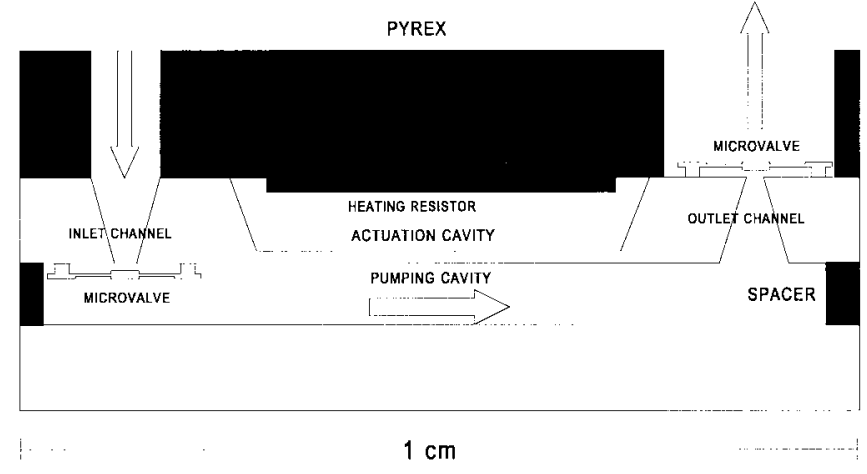

Fig. 3. Thermo-pneumatic actuated micropump.

careful choice of the differentiator can mitigate this effect and in consequence it permits higher time resolutions.

\section{APPLICATIONS}

\section{A. Physical Simulation}

The method has been first applied to dynamic 3-D thermal FEM simulations of an actuation unit belonging to a thermopneumatic actuated micropump (Fig. 3) [4].

ANSYS 5.3 has been used for these physical simulations. A step power is applied to the heating resistor and the temperature increment of the air inside the actuation cavity can be obtained. Simulation time points have been constant logarithmically distributed. It was decided to take twenty points per time decade along six decades; that is to say, 120 FEM solution steps were calculated. The temperature increment obtained by this way can be seen in Fig. 4.

The application of the method to the former thermal impedance results in a three peaks function, shown in Fig. 5. The result is compared to the simple differentiator used by Szekely [11] (first order METS signal).

The three time constants and exponential amplitudes associated with these peaks are obtained by nonlinear fitting. 


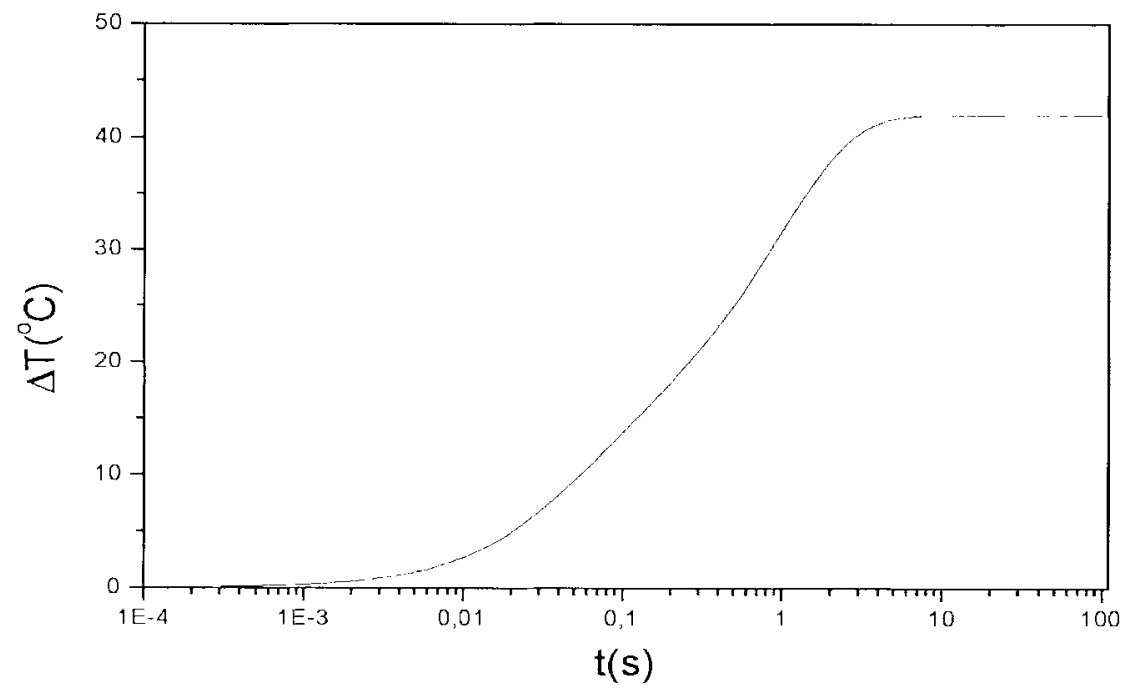

Fig. 4. Thermal impedance of the thermo-pneumatic micropump from FEM analysis.

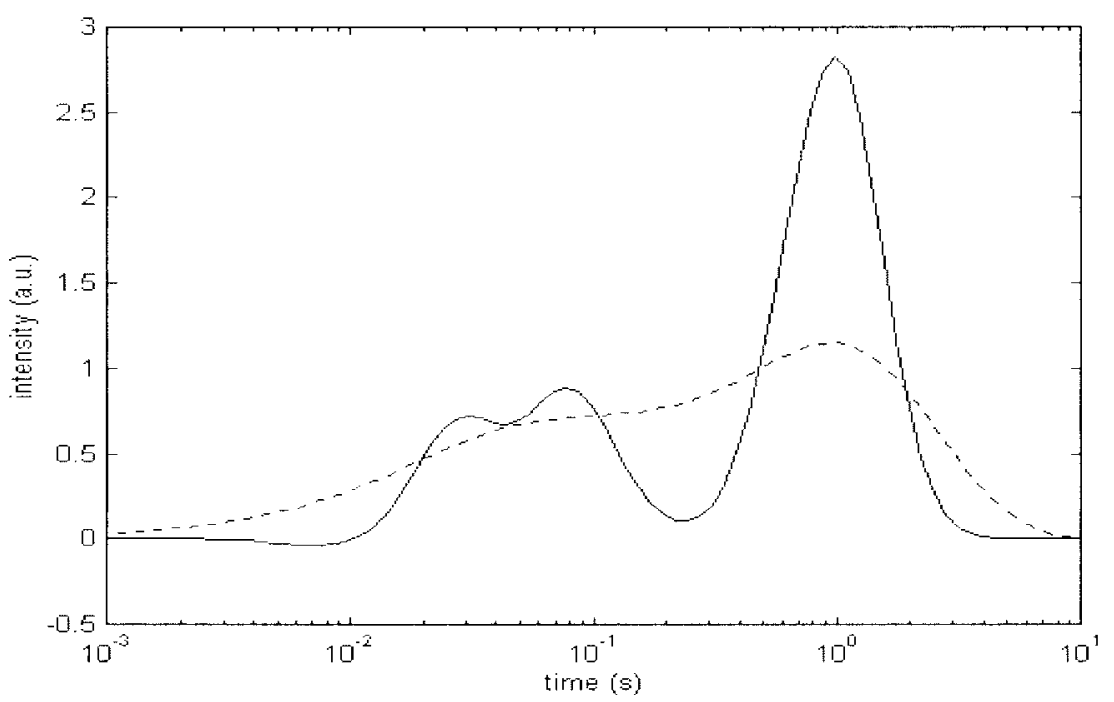

Fig. 5. First (dotted) and tenth (line) order METS applied to the transient of Fig. 4.

TABLE I

Transfer Function PARAMETERS

\begin{tabular}{c|c|c}
\hline & Time constant(s) & Amplitude( ${ }^{(1 \mathrm{C} / \mathrm{W})}$ \\
\hline 1 & 0.023 & 17.6 \\
2 & 0.062 & 21.5 \\
3 & 0.740 & 69.9 \\
\hline
\end{tabular}

They are listed in Table I. Although we suspect that these three time constants are associated to the presence of three different materials in the model (pyrex, air and silicon), the identification of the Cauer RC values is not trivial due to the existence of 3-D heat path flows. Differences of 1.35 octaves in time constant can be resolved in this particular case. However, with synthetic transients with added relative noise amplitude of $10^{-8}$ the technique is able to separate 0.84 octaves, increasing the METS order till 20.

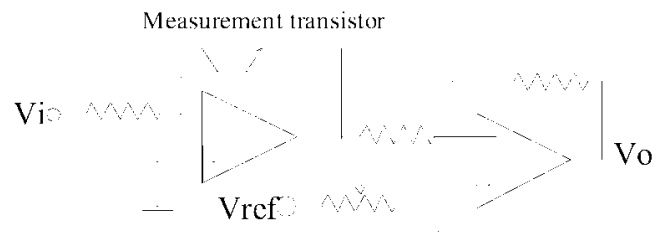

Fig. 6. VBE measurement circuit.

\section{B. Application to Experimental Measurements}

More difficulties can be encountered in applying the method to experimental results. There exists some previous works related to the measurement of thermal impedance. Zweidinger et al. [18] use the same device (a bipolar transistor) to apply the power and to measure the temperature. In this study, the temperature measurement is based on the base current variations keeping the base-emitter voltage constant. Also, Assouad et al. [19] use thermal impedance measurement for package modeling by using the Double Cold Plated measurement setup. 


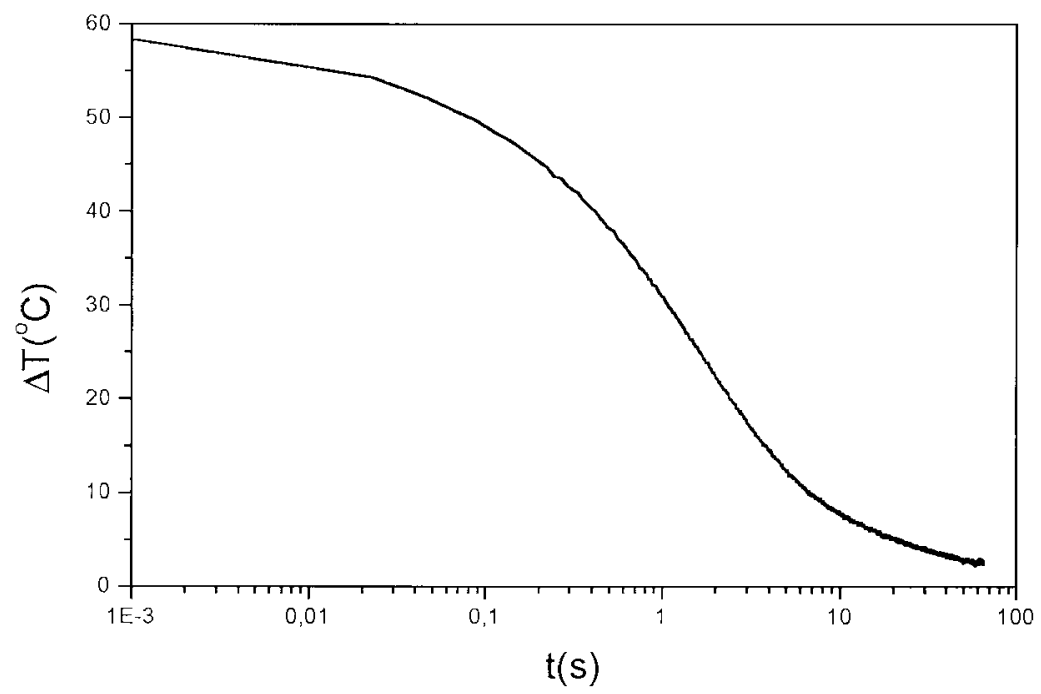

Fig. 7. Transient thermal impedance of the HFA 3096 IC.

This method assumes a constant temperature over the device. Christiaens [1] use the submerged double jet impingement (SDJI) method in order to obtain a better controlled boundary condition. Temperature is measured using a diode, or bipolar transistor, under forward biasing.

In our case, we have applied our method to the analysis of the thermal impedance of the IC HFA 3096 with a small outline plastic package (SOIC), from Harris Semiconductor's. It consists of an array of five dielectrically isolated transistors, three npn and two pnp. Monolithic construction allows a close electrical and thermal matching between these transistors. In this case, we will use only two of the npn transistors. A heat sink with a good thermal contact with one of the principal faces has been used to fix the temperature in that face and the other main surface is supposed to be thermally isolated; this procedure results in the speeding up of the transient with respect air boundary conditions. This experimental setup is called temperature controlled heat-sink method [20]. One of the npn transistors is used to apply a power step to the chip. In order to obtain a constant power dissipation during the transient, we apply a negative power step of $0.53 \mathrm{~W}$. Once steady state conditions are reached, base current in the heater is switched off. In this way, we avoid the problems that appear in positive power steps due to the dependence of $\beta$ with the temperature. As opposed to [18], a neighbor transistor connected as a diode is supplied with a constant current $1.5 \mathrm{~mA}$ and it is used to sense the temperature via its $V_{B E}$ variations.

The circuit used in this work to extract the junction temperature can be seen in Fig. 6. The measurement transistor is supplied with a constant $I_{B}$ current. The first operational amplifier is used to extract the $V_{B E}$ of the transistor. The second one provides the amplified $\Delta V_{B E}$ voltage value. This output voltage is acquired with a Lab-PC + acquisition board installed in a PC. Signal acquisition is triggered by the power step.

In this section we will apply the METS method to the experimental data. As a reference we will use the truncated
Van-Citter deconvolution. For a description of this method see [15]. Fig. 7 shows the measured temperature evolution of the chip in the logarithmic time domain after a negative power step. A time-varying filter is applied to transform the original linear time-scale to the logarithmic time-scale needed to perform the METS transformation. The first order METS signal of the thermal transient is shown in Fig. 8.

The modified truncated Van-Citter deconvolution method gives a highly changing time-constant-spectrum during its iterative evolution. With this time constant spectrum (at any iteration), it is possible to generate the first order METS signal and then evaluate the mean square error between the recovered signal and the measured one. Minimization of this objective function has been taken as criterion to decide the optimum number of iterations during the Van-Citter deconvolution.

Fig. 9 is the time-constant spectrum of the chip-caseambient thermal structure obtained with a modified Truncated Van-Citter deconvolution method applied to the first order METS signal. 1596 iterations were needed. In the same figure, 4th order METS is also plotted. A good agreement is obtained between very different methods. It seems that a continuous time-constant distribution can explain the obtained result. Alternatively, a set of close enough time constants can deliver a similar result. In this case the METS would provide a low-pass filtered version of the time-constant spectrum. When compared to the truncated Van-Citter deconvolution, it can be noted that the METS method requires much less computation power.

\section{CONCLUSION}

Thermal impedance analysis is a powerful tool to extract the thermal dynamics of chip packages or thermally based microsystems. However a careful analysis of the transient is mandatory to extract the underlying time-constant spectrum which contains all the information of the corresponding transfer function. This analysis becomes hard because of the nonorthogonality of real exponentials. The METS method has 


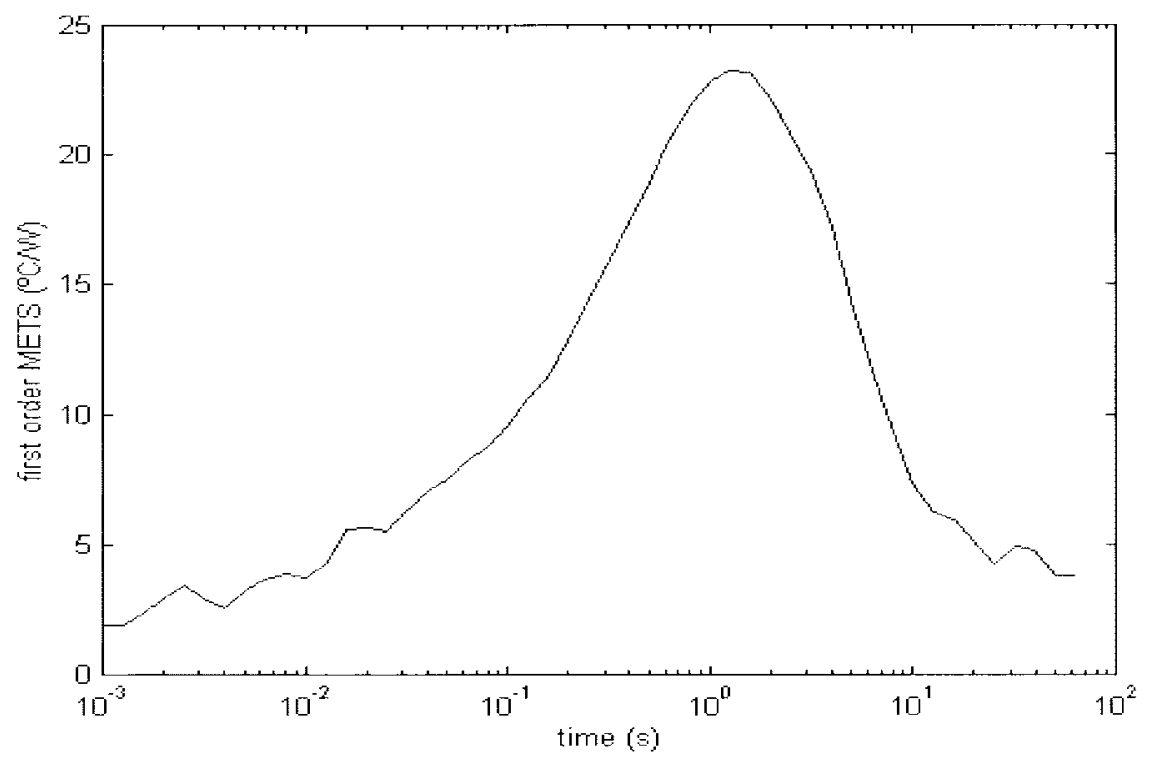

Fig. 8. 1st order METS for the thermal transient.

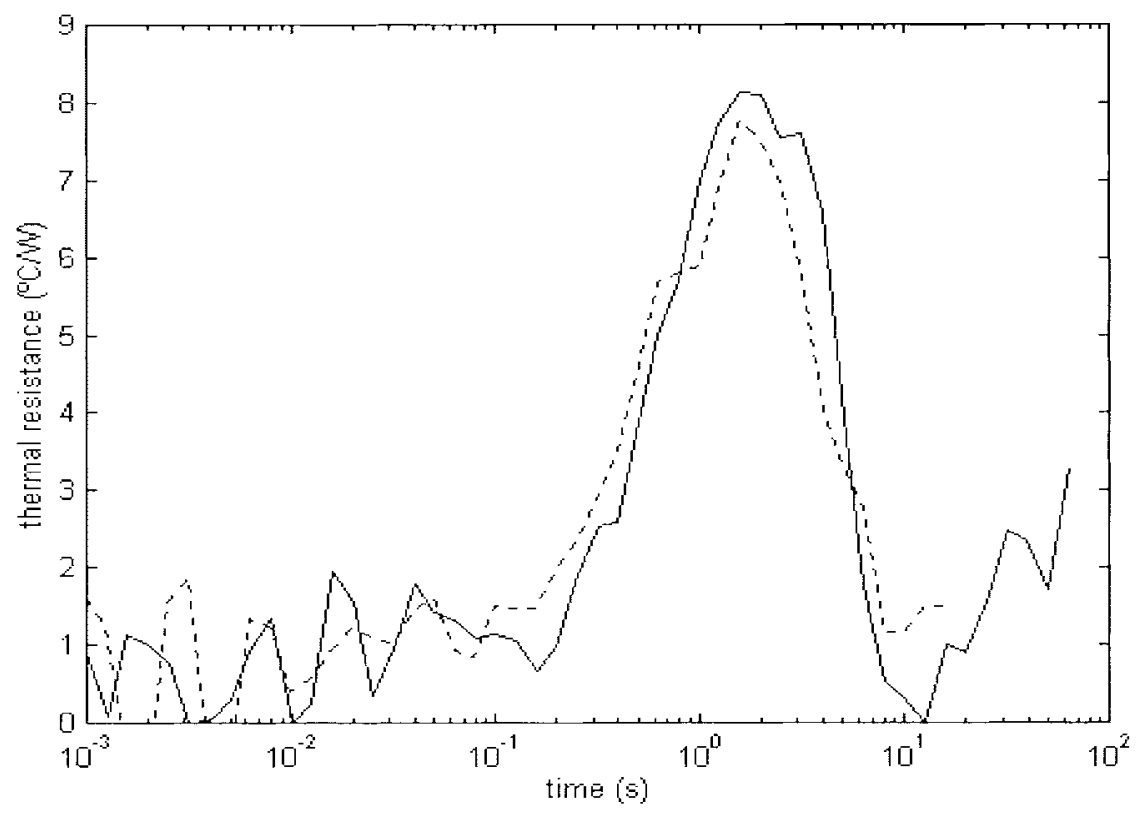

Fig. 9. Comparison between the time-spectrum obtained by the truncated Van-Citter deconvolution (solid line) and 4th order METS.

been presented and it has been applied to both simulated and empirical transients. A great increase in resolution is obtained with minimum computational effort. In the analysis of experimental transients the noise level limits the time resolution to levels which are comparable to those of the truncated Van-Citter method. Further work is needed to characterize the performance limits of the proposed method. This method implies a noteworthy improvement in the extraction of compact thermal models from experimental or simulated data.

\section{ACKNOWLEDGMENT}

The authors would like to thank all the reviewers for their valuable suggestions.

\section{REFERENCES}

[1] I. F. Christiaens, "Thermal modeling and characterization of electronic components: Steady-state and transient analysis," Ph.D. dissertation, Katholieke Univ. Leuven, Belgium, 1998.

[2] Y. Pan, F. R. Riedijk, and J. H. Huijsing, "A new class of integrated thermal oscillators with duty-cycle output for application in thermal sensors," Sens. Actuators A, vol. 21-23, pp. 655-659, 1990.

[3] C. A. Leme and H. Baltes, "Interfaces for microsensor systems" in W. Sansen et al. Analog Circuit Design. Norwell, MA: Kluwer, 1994, pp. 163-181.

[4] S. Marco, M. Carmona, and J. Samitier, "Extraction of HDL-A models of thermally based microsystems from physical simulations," in Proc. Int. Conf. Simulation Sensors Microsyst. MSM98, St. Clara, CA, Apr. 1998.

[5] G. Krieger, "Thermal response of integrated circuit input devices to an electrostatic energy pulse," IEEE Trans. Electron Devices, vol. ED-34, 1987. 
[6] F. Christiaens, B. Vandevelde, E. Beyne, and J. Roggen, "Evaluation of structural degradation in packaged semiconductor components using a transient thermal characterization technique," Microelectron. Rel., vol. 36, pp. 1807-1810, 1996.

[7] J. W. Sofia, "Analysis of thermal transient data with synthesized dynamic models for semiconductor devices," IEEE Trans. Comp., Packag., Manufact. Technol. A, vol. 18, pp. 39-47, Mar. 1995.

[8] C. T. Fike, "Computer Evaluation of Mathematical Functions." Englewood Cliffs, NJ: Prentice-Hall, 1968.

[9] A. Piccirillo, G. Oliveti, M. Ciampa, and P. E. Bagnoli, "Complete characterization of laser diode thermal circuit by voltage transient measurements," Electron. Lett., vol. 29, pp. 318-320, 1993.

[10] M. R. Osbourne, "Some special nonlinear least square problems," SIAM J. Numer. Anal., vol. 12, pp. 571-592, 1975.

[11] V. Szekely and T. V. Bien, "Fine-structure of heat flow path in semiconductor devices: A measurement and identification method," Solid-State Elecron., vol. 31, pp. 1363-1368, 1988.

[12] D. G. Gardner, J. C. Gardner, G. Lush, and W. W. Meinke, "Method for the analysis of multicomponent exponential decay curves," J. Chem. Phys., vol. 31, pp. 978-986, 1959.

[13] M. R. Smith, S. Cohn-Sfetcu, and H. A. Buckmaster, "Decomposition of multicomponent exponential decays by spectral analytic techniques," Technometrics, vol. 18, pp. 467-482, 1976.

[14] V. Szekely, "Identification of RC networks by deconvolution: Chances and limits," IEEE Trans. Circuits Syst., vol. 45, pp. 244-258, 1998.

[15] P. A. Jansson, Deconvolution of Images and Spectra. San Diego, CA: Academic, 1997.

[16] S. Marco. J. Palacín, A. Guibernau, and J. Samitier, "Improved multiexponential transient spectroscopy by iterative deconvolution," in Proc. IMTC98 Instrum. Meas. Technol. Conf., St. Paul, MN, May 1998, pp. 670-674.

[17] S. Marco, J. Samitier, and J. R. Morante, "A novel time-domain method to analyze multicomponent exponential transients," Meas. Sci. Technol., vol. 6, pp. 135-142, 1995

[18] D. T. Zweidinger, R. M. Fox, J. S. Brodsky, T. Jung, and S.-G. Lee, "Thermal impedance extraction for bipolar transistors," IEEE Trans. Electron Devices, vol. 43, no. 2, pp. 342-346, 1996.

[19] Y. Assouad, F. Gatfosse, and T. Gautier, "Transient characterization and modeling of ceramic packages," in Proc. Int. Workshop Thermal Investigat. IC's Microstruc., (THERMINIC'96), 1996, pp. 243-252.

[20] SEMI Standard G30-88, "Test method junction-to-case thermal resistance measurements of ceramic packages," Book of SEMI Standards, 1995.

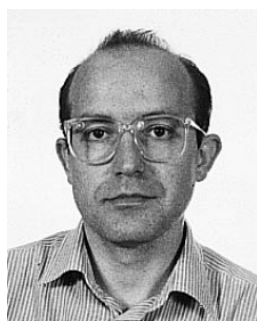

Manuel Carmona received the degree in physics from the Universitat de Barcelona, Barcelona, Spain, in 1993, where he is currently pursuing the Ph.D. degree in the Department of Electronics.

His main interests are microsystems modeling, and design by finite element methods and analog behavioral models.

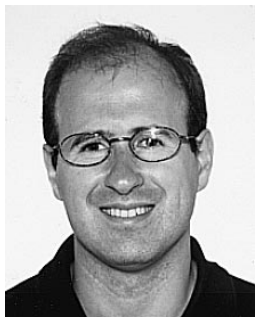

Santiago Marco received the B.S. degree in physics and the Ph.D. degree (with honors) for the development of a novel silicon sensor for in-vivo measurements of the blood pressure, both from the Universitat de Barcelona, Barcelona, Spain, in 1988 and 1993, respectively.

He has been an Associate Professor at the Universistat de Barcelona since 1995. From 1989 to 1990, he worked in the electro-optical characterization of deep levels in GaAs. From 1990 to 1993, he was a regular visitor to the Centro Nacional de Microelectrònica. In 1994, he was a Visiting Professor at the "Tor Vergata," Universita di Roma, Rome, Italy, working in electronic noses. He has published over 25 papers in sciencific journals and books. His current research interests are the modeling, simulation and test of microsystems and the development of intelligent gas sensor arrays.

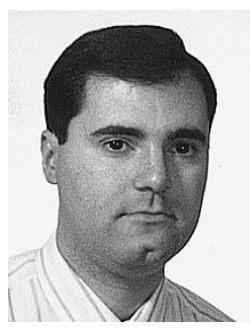

Jordi Palacín received the degree in electronic engineering from the Universitat de Barcelona, Barcelona, Spain, in 1997 where he is currently pursuing the Ph.D. degree in the Departament of Electronics.

$\mathrm{He}$ is an Associate Professor in the Departament d'Informàtica i Enginyeria Industrial, Universitat de Lleida, Spain. His research interests include signal processing, sensors, and robotics.

Josep Samitier received the degree in physics from the University of Barcelona, Barcelona, Spain, in 1982.

He has been a Full Professor at the University of Barcelona since 1995. He was a Research Fellow from 1983 to 1984 in the Applied Physics Department, University of Barcelona, in the field of GaAs MESFET devices and electrooptical characterization of III-V semiconductors. From February 1984 to June 1985, he was a Visiting Research Fellow at the Laboratoire D'Electronique Philips, LEP, Paris, France. In 1988, he was appointed Associate Professor to work in the development of microsystems and electronic instrumentation. Current research and developed projects concern the design, test, and signal conditioning of microsystems and the design of interface circuits for sensors and microsystems. At the present, he coordinates the projects in electronic instrumentation of the Electronic Engineering R\&D activities. 